\title{
On a discrete-time model with replicator dynamics in renewable resource exploitation
}

\author{
Gian Italo Bischi \\ Universitità di Urbino Carlo Bo \\ gian.bischi@uniurb.it
}

\author{
Lorenzo Cerboni Baiardi \\ Università di Urbino Carlo Bo \\ l.cerbonibaiardi@campus.uniurb.it
}

\author{
Davide Radi \\ Università Politecnica delle Marche \\ d.radi@univpm.it
}

June 3, 2015

\begin{abstract}
We consider a discrete time version of the model proposed by Lamantia and Radi (2015) to describe a fishery where a population regulated by a logistic growth function is exploited by a pool of agents that can choose, at each time period, between two different harvesting strategies according to a profit-driven evolutionary selection rule. The resulting discrete dynamical system, represented by a two-dimensional nonlinear map, is characterized by the presence of invariant lines on which the dynamics are governed by one-dimensional restrictions that represent pure, i.e. adopted by all players, strategies. However, interesting dynamics related to interior attractors, where players playing both strategies coexist, are evidenced by analytical as well as numerical methods that reveal local and global bifurcations.
\end{abstract}

Keywords: discrete-time population model, replicator dynamics, resource exploitation, attractors, bifurcations.

\section{Introduction}

In this paper we consider a discrete time version of the model proposed in [15] to describe a fishery where two different harvesting strategies can be employed, one denoted as standard and the other one more ecological (less intensive, hence more environmentally friendly). At any time period, the fish population is assumed to reproduce according to a discrete-time logistic growth function (see e.g. [7] chapter 2 and [13]), and the agents that exploit the fishery are assumed to update their harvesting strategy according to a profit-driven adaptive mechanism based on the evolutionary selection rule known as replicator dynamics (see e.g. [20] and [14]).

Even if dynamic models in ecology have been traditionally formulated in continuous time, discrete-time population models have received a great amount of attention not only for the complex 
and intriguing dynamics that they can produce even in the simplest systems, but also because biological motivations have been proposed to explain their usefulness in ecologic modeling. In fact, several authors stress that discrete-time population models should be used whenever reproduction happens at given breeding seasons, as several animal species successfully mate only during certain times of the year, thus giving non-overlapping generations (see e.g. [12] and [17]). So, more and more discrete-time population models have been proposed in the literature (see e.g. [13], [5] and [10]). Moreover, as already stressed in [15], in the model considered in this paper decisions about the kind of harvesting strategy to be adopted typically occur in discrete time, as such decisions imply the adoption of different fishing technologies, and/or different numbers of workers with different kinds of equipment, hence they cannot be revised at any time. Sometimes the possibility of switching from a harvesting strategy to another one is allowed only at given time periods by laws that regulate harvesting activities (see [4], [3] and [6]).

On the basis of these motivations, a discrete-time model, represented by a two-dimensional nonlinear map, is studied in this paper by using analytical, geometric and numerical methods. The structure of the map is quite interesting from the point of view of its mathematical properties, and is typical (hence representative) for a large class of repeated evolutionary games where a population of $N$ players can choose, at any time, between two strategies. In fact, one dynamic variable, denoted as $r(t) \in[0,1]$, represents the fraction of players adopting a given strategy at time period $t \in \mathbb{N}$ (of course the complementary fraction $1-r(t)$ adopts the other strategy at the same time period). As typically occurs in these kind of evolutionary games, the two lines $r=0$ and $r=1$, where all players adopt the same strategy, are invariant lines, along which the dynamics characterized by unique kind of players (pure strategy case) are governed by a onedimensional restriction of the map. In our case, the dynamics along such invariant boundary lines are given by the iteration of a quadratic map, topologically conjugate to the standard logistic map. However, interior attractors, where players carrying out both strategies coexist, can be obtained, and some bifurcations involving interior and boundary invariant sets can be studied. Indeed, very rich dynamic scenarios can be highlighted, both analytically and numerically, and regions of the phase space of the model can be detected in which quasi-periodic motions prevail (i.e. where the linear approximation of the map has complex eigenvalues) and other regions where real eigenvalues give rise to monotonic motions or improper oscillations. In both cases, however, transitions to chaotic behaviors can be observed. Moreover, the existence of non topological Milnor attractors embedded in the invariant lines is proved and numerically shown.

The paper is organized as follows. Section 2 presents the discrete-time model and discusses the dynamics along the invariant lines where one-dimensional dynamics occurs when all agents adopt the same strategy. Section 3 contains analytical results on the existence of equilibrium points and their local stability properties as well as local bifurcations. Section 4 gives some propositions on global behavior and some numerical simulations of the model. Section 5 concludes and indicates further research issues.

\section{The model}

Following the general setup of the model proposed by [15], let $x(t)$ denote the available quantity at time $t$ of a renewable resource and let us consider a population of $N$ agents that can exploit the resource by two different technologies: a standard (intensive) one characterized by technology 
coefficient $q_{1}>0$, and a more environmentally-friendly (let's say ecological) technology characterized by $q_{0} \in\left(0, q_{1}\right)$. Let $r(t) \in[0,1]$ be the fraction of agents that adopt the standard technology during time period $t$ and consequently the complementary fraction of agents $(1-r(t))$ adopts the ecological technology, so that $r=0$ means that all the agents adopt the ecological technology $q_{0}$, and $r=1$ means that all the agents adopt the standard technology $q_{1}$. If $h_{i}$ denotes the harvesting of resource by using technology $i, i=0,1$, following again [15] we assume that the cost functions are given by

$$
C_{i}\left(h_{i}\right)=c_{i}+\gamma \frac{h_{i}^{2}}{q_{i} x} ; i=0,1
$$

where $c_{i} \leq 0$ represents fixed costs and $\gamma>0$ is a cost coefficient.

If we denote by $a_{0}>0$ the constant price at which consumers buy the resource harvested by ecological technology and $a_{1} \in\left(0, a_{0}\right)$ the price at which they buy the standard one, then, as shown in [15], the optimal harvesting, computed as Nash equilibrium, of the representative player that uses technology $i$ is given by

$$
h_{i}(x)=\frac{a_{i} q_{i}}{2 \gamma} x ; i=0,1
$$

In the following $a_{0}>a_{1}$ will be assumed, i.e. the loss in efficiency of the more ecological harvesting strategy is counterbalanced by a higher price that consumers wish to pay for the more environmentally-friendly product.

Differently from [15], we consider a discrete-time model, i.e. a resource is given by a population with non-overlapping generations growing according to a discrete-time logistic equation

$$
x(t+1)=x(t)+\alpha x(t)\left(1-\frac{x(t)}{k}\right)-N r(t) h_{1}(t)-N(1-r(t)) h_{0}(t)
$$

Here $\alpha>0$ is the natural growth rate of the resource, $k>0$ represents the carrying capacity, i.e. the equilibrium level of the resource in the absence of harvesting, and fraction $r(t)$ is assumed to evolve according to an exponential replicator dynamics (see e.g. [9] and [14]) driven by profits

$$
\pi_{i}(x)=a_{i} h_{i}(x)-c_{i}-\gamma \frac{h_{i}^{2}(x)}{q_{i} x}
$$

and expressed by

$$
r(t+1)=\frac{r(t) e^{\beta \pi_{1}(t)}}{r(t) e^{\beta \pi_{1}(t)}+(1-r(t)) e^{\beta \pi_{0}(t)}}=\frac{r(t)}{r(t)+(1-r(t)) e^{\beta(\Delta \pi(t))}}
$$

where $\beta \in[0,+\infty)$ is the so called intensity of choice parameter and measures the reactiveness of agents to adopt the more profitable strategy and $\Delta \pi(t)$ is the difference between the two profits. According to (4) and (2), we have that

$$
\Delta \pi(t)=\pi_{0}(t)-\pi_{1}(t)=\frac{a_{0}^{2} q_{0}-a_{1}^{2} q_{1}}{4 \gamma} x(t)-\xi
$$

with $\xi=c_{0}-c_{1}$. The parameter $\xi \in \mathbb{R}$ represents the difference between fixed costs associated with the two technologies, and may be considered as a policy parameter as it includes taxes imposed in 
order to obtain the prevalence of one technology over the other. In the following we shall mainly consider $\xi \leq 0$, assuming that fixed costs for the more intensive harvesting method are higher, due to more sophisticated technology and higher taxes, or equivalently to government subsidies for agents adopting the more ecological fishing methods.

All in all, the dynamic model can be written in the form of an iterated map of the plane $T:(x, y) \rightarrow\left(x^{\prime}, y^{\prime}\right)$ with

$$
T:\left\{\begin{array}{l}
x^{\prime}=\left(1+\alpha-\frac{N a_{0} q_{0}}{2 \gamma}\right) x-\frac{\alpha}{k} x^{2}+\frac{N}{2 \gamma}\left(a_{0} q_{0}-a_{1} q_{1}\right) x r \\
r^{\prime}=\frac{r}{r+(1-r) e^{\beta\left(\frac{a_{0}^{2} q_{0}-a_{1}^{2} q_{1}}{4 \gamma} x-\xi\right)}}
\end{array}\right.
$$

where' denotes the unit-time advancement operator, and the dynamic variables represent feasible states of the system if $x \geq 0$ and $0 \leq r \leq 1$.

It is worth underlining that the line of resource extinction $x=0$, as well as the two lines of pure strategies $r=0$ and $r=1$, are invariant sets. The dynamics along the invariant line $x=0$ is governed by the one-dimensional restriction

$$
x=0: \quad r^{\prime}=g(r)=\frac{r}{r+(1-r) e^{-\xi}}
$$

which is a convex function in the interval $r \in[0,1]$ with fixed points in $r=0$ (stable) and $r=1$ (unstable). The dynamics along the invariant line $r=0$, where all the agents adopt the ecological strategy, are governed by the one-dimensional restriction

$$
r=0: \quad x^{\prime}=f_{0}(x)=\left(1+\alpha-\frac{N a_{0} q_{0}}{2 \gamma}\right) x-\frac{\alpha}{k} x^{2}
$$

topologically conjugate to the standard logistic map $z^{\prime}=\mu z(1-z)$ by the transformation $z=$ $\frac{2 \gamma \alpha}{k\left[2 \gamma(1+\alpha)-N a_{0} q_{0}\right]} x$ and parameter $\mu=1+\alpha-\frac{N a_{0} q_{0}}{2 \gamma}$. Its two fixed points are given by $x_{0}^{0}=0$ (extinction equilibrium) and

$$
x_{0}^{*}=\frac{k\left(2 \alpha \gamma-N a_{0} q_{0}\right)}{2 \alpha \gamma}
$$

that represents the viable equilibrium under ecological harvesting. Notice that $x_{0}^{*}$ is stable for the dynamics along the line $r=0$ provided that

$$
\alpha-2<\frac{N a_{0} q_{0}}{2 \gamma}<\alpha
$$

where the condition $\frac{N a_{0} q_{0}}{2 \gamma}=\alpha$ represents the transcritical bifurcation along the line $r=0$ at which the viable equilibrium $x_{0}^{*}$ merges with the extinction equilibrium $x_{0}^{0}$, whereas the condition $\frac{N a_{0} q_{0}}{2 \gamma}=\alpha-2$ represents a period doubling bifurcation, at which a stable cycle becomes the unique attractor along the line $r=0$. As it is well known, this bifurcation opens the period-doubling cascade, leading to chaotic motion along the line $r=0$, as the aggregate parameter $\frac{N a_{0} q_{0}}{2 \gamma}$ is further decreased. 
Analogously, along the invariant line $r=1$ where all agents adopt the standard (more intensive) fishing strategy, the dynamics are governed by the map

$$
r=1: \quad x^{\prime}=f_{1}(x)=\left(1+\alpha-\frac{N a_{1} q_{1}}{2 \gamma}\right) x-\frac{\alpha}{k} x^{2}
$$

conjugate to the logistic map $z^{\prime}=\mu z(1-z)$ by the transformation $z=\frac{2 \gamma \alpha}{k\left[2 \gamma(1+\alpha)-N a_{1} q_{1}\right]} x$ and parameter $\mu=1+\alpha-\frac{N a_{1} q_{1}}{2 \gamma}$. Here, the viable equilibrium is

$$
x_{1}^{*}=\frac{k\left(2 \alpha \gamma-N a_{1} q_{1}\right)}{2 \alpha \gamma}
$$

Notice $x_{0}^{*}>x_{1}^{*}$ if $a_{0} q_{0}<a_{1} q_{1}$, a condition that we shall assume in the following in order to characterize the technology $q_{0}$ as more ecological. As for $x_{0}^{*}$, stability conditions of the viable equilibrium along the line $r=1$ can be obtained by straightforward calculations, and are given by

$$
\alpha-2<\frac{N a_{1} q_{1}}{2 \gamma}<\alpha
$$

where similar statements about the transcritical and period doubling bifurcations hold.

The existence of these invariant lines that bound the two-dimensional phase space of the dynamical system (7) is important in order to characterize its global dynamical properties. Moreover, the knowledge of the kind of dynamic motion occurring along the two lines where a single pure strategy exists, tell us what will happen in the long run when one of the two strategies becomes dominant in terms of profits so that it will prevail due to evolutionary pressure. The latter problem may be equivalently stated by asking when the one-dimensional attractors of the restrictions along the invariant lines $r=0$ and $r=1$ given by (9) and (12) respectively, are also attractors of the two-dimensional dynamical system. This depends on the transverse stability as well as on the existence of attractors interior to the phase space, i.e. characterized by $r \in(0,1)$. These are the questions examined, analytically and numerically, in the next sections. Here, for the sake of clarity, it is worth specifying that the $\mathrm{j}$-cycle $(\mathrm{j} \geq 1)$ laying on an invariant line has one of its eigenvectors that is along the invariant line itself, while the other eigenvector has generally another direction. This last eigenvector is commonly named transverse eigenvector, which is tangent to the so-called transverse invariant manifold.

\section{$3 \quad$ Existence and stability of equilibrium points}

The equilibrium points of the model (7) are solutions of the system

$$
\left\{\begin{array}{l}
x\left[\alpha-\frac{N a_{0} q_{0}}{2 \gamma}-\frac{\alpha}{k} x+\frac{N}{2 \gamma}\left(a_{0} q_{0}-a_{1} q_{1}\right) r\right]=0 \\
r(1-r)\left[e^{\beta\left(\frac{a_{0}^{2} q_{0}-a_{1}^{2} q_{1}}{4 \gamma} x-\xi\right)}-1\right]=0
\end{array}\right.
$$

The extinction fixed points $E_{0}^{0}=(0,0)$ and $E_{1}^{0}=(0,1)$ always exist. Moreover, if $\xi=0$ then any point of the whole segment $(0, r)$, with $r \in[0,1]$, is a fixed point. Other boundary equilibrium 
points are $E_{0}^{*}=\left(x_{0}^{*}, 0\right)$ with $x_{0}^{*}$ given by $(10)$ and $E_{1}^{*}=\left(x_{1}^{*}, 1\right)$ with $x_{1}^{*}$ given by $(13)$. Furthermore, an interior equilibrium may exist, characterized by the co-existence of both harvesting strategies, given by $E^{*}=\left(x^{*}, r^{*}\right)$ with

$$
x^{*}=\frac{4 \gamma \xi}{a_{0}^{2} q_{0}-a_{1}^{2} q_{1}} ; \quad r^{*}=\frac{2 \alpha \gamma\left(k-x^{*}\right)-N k a_{0} q_{0}}{N k\left(a_{1} q_{1}-a_{0} q_{0}\right)}
$$

provided that $x^{*}>0$ and $r^{*} \in(0,1)$.

Let us notice that if $\xi<0$, i.e. $c_{0}<c_{1}$ as argued above, then $x^{*}>0$ provided that $a_{0}^{2} q_{0}<a_{1}^{2} q_{1}$, which is a more stringent condition than $a_{0} q_{0}<a_{1} q_{1}$ being $a_{0}>a_{1}$. We shall assume that this condition is satisfied in the following ${ }^{1}$. It is worth noticing that the condition $r^{*} \in(0,1)$ can be easily expressed in term of the carrying capacity $k$, as $r^{*}=0$ for $k=k_{0}$ with

$$
k_{0}=\frac{2 \alpha \gamma x^{*}}{2 \alpha \gamma-N a_{0} q_{0}}=\frac{8 \alpha \gamma^{2} \xi}{\left(2 \alpha \gamma-N a_{0} q_{0}\right)\left(a_{0}^{2} q_{0}-a_{1}^{2} q_{1}\right)}
$$

and $r^{*}=1$ for $k=k_{1}$ with

$$
k_{1}=\frac{2 \alpha \gamma x^{*}}{2 \alpha \gamma-N a_{1} q_{1}}=\frac{8 \alpha \gamma^{2} \xi}{\left(2 \alpha \gamma-N a_{1} q_{1}\right)\left(a_{0}^{2} q_{0}-a_{1}^{2} q_{1}\right)}
$$

with $k_{0}<k_{1}$ being $a_{0} q_{0}<a_{1} q_{1}$, so that $r^{*} \in(0,1)$ for $k_{0}<k<k_{1}$.

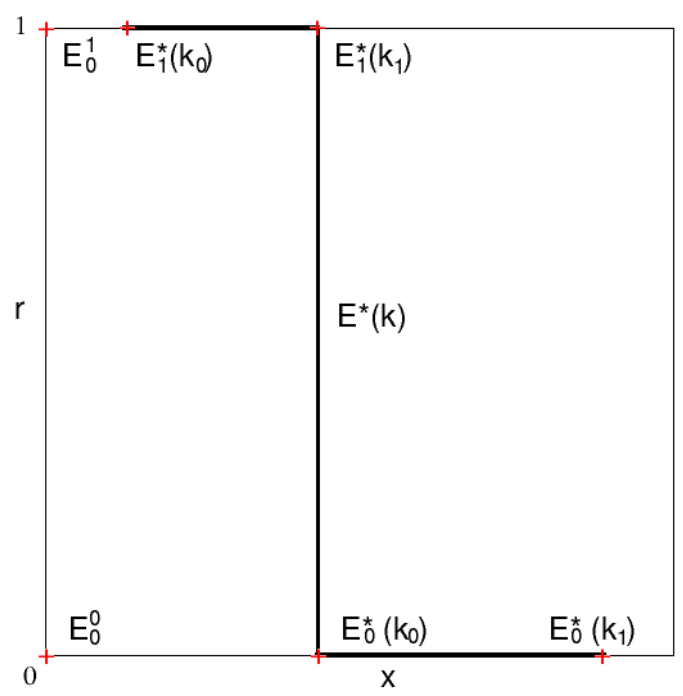

Figure 1: Fixed points $E^{*}(k), E_{0}^{*}(k)$ and $E_{1}^{*}(k)$ are shown as $k \in\left[k_{0}, k_{1}\right]$. The point $E^{*}$ merges with $E_{0}^{*}$ in $r=0$ for $k=k_{0}$ and merges with $E_{1}^{*}$ in $r=1$ for $k=k_{1}$. Other fixed points do not depend on $k$.

These existence conditions are the same as the ones given in [15] for the model in continuous time, whereas the stability conditions now are different. In order to study the local stability of

\footnotetext{
${ }^{1}$ For sake of completeness, we stress that if $\xi>0$, i.e. $c_{0}>c_{1}$, then $x^{*}>0$ provided that $a_{0}^{2} q_{0}>a_{1}^{2} q_{1}$, that together with the condition $a_{0} q_{0}<a_{1} q_{1}$ gives $\frac{a_{1}^{2} q_{1}}{a_{0}}<a_{0} q_{0}<a_{1} q_{1}$, which is a nonempty set being $a_{0}>a_{1}$.
} 
the equilibrium points we consider the Jacobian matrix

$$
J(x, r)=\left[\begin{array}{cc}
1+\alpha-\frac{N a_{0}}{2 \gamma}-\frac{2 \alpha}{k} x-\frac{N}{2 \gamma}\left(a_{1} q_{1}-a_{0} q_{0}\right) r & -\frac{N\left(a_{1} q_{1}-a_{0} q_{0}\right) x}{2 \gamma} \\
\frac{\beta r(1-r)\left(a_{1}^{2} q_{1}-a_{0}^{2} q_{0}\right) e^{\beta \Delta \pi}}{4 \gamma\left(r+(1-r) e^{\beta \Delta \pi}\right)^{2}} & \frac{e^{\beta \Delta \pi}}{\left(r+(1-r) e^{\beta \Delta \pi}\right)^{2}}
\end{array}\right]
$$

At $E_{0}^{0}$ we have

$$
J(0,0)=\left[\begin{array}{cc}
1+\alpha-\frac{N a_{0} q_{0}}{2 \gamma} & 0 \\
0 & e^{\beta \xi}
\end{array}\right]
$$

so $E_{0}^{0}$ is stable along the vertical direction ( $r$ direction) and stable along the horizontal one $(x$ direction) provided that $\frac{N a_{0} q_{0}}{2 \gamma}-2<\alpha<\frac{N a_{0} q_{0}}{2 \gamma}$. At $E_{1}^{0}$ we have

$$
J(0,1)=\left[\begin{array}{cc}
1+\alpha-\frac{N a_{1} q_{1}}{2 \gamma} & 0 \\
0 & e^{-\beta \xi}
\end{array}\right]
$$

so $E_{1}^{0}$ is unstable along the vertical direction and stable along the horizontal one provided that $\frac{N a_{1} q_{1}}{2 \gamma}-2<\alpha<\frac{N a_{1} q_{1}}{2 \gamma}$. At $E_{0}^{*}$ we have

$$
J\left(x_{0}^{*}, 0\right)=\left[\begin{array}{cc}
1-\alpha+\frac{N a_{0} q_{0}}{2 \gamma} & -\frac{N\left(a_{1} q_{1}-a_{0} q_{0}\right)\left(2 \alpha \gamma-N a_{0} q_{0}\right)}{2 \alpha \gamma^{2}} \\
0 & e^{\beta\left(\frac{k\left(a_{1}^{2} q_{1}-a_{0}^{2} q_{0}\right)\left(2 \alpha \gamma-N a_{0} q_{0}\right)}{8 \alpha \gamma^{2}}+\xi\right)}
\end{array}\right]
$$

so $E_{0}^{*}$ is stable along the eigendirection transverse to $r=0$ if $k<k_{0}$, with $k_{0}$ given by (17), and stable along the horizontal direction if $\frac{N a_{0} q_{0}}{2 \gamma}<\alpha<\frac{N a_{0} q_{0}}{2 \gamma}+2$.

At $E_{1}^{*}$ we have

$$
J\left(x_{1}^{*}, 1\right)=\left[\begin{array}{cc}
1-\alpha+\frac{N a_{1} q_{1}}{2 \gamma} & -\frac{N\left(a_{1} q_{1}-a_{0} q_{0}\right)\left(2 \alpha \gamma-N a_{1} q_{1}\right)}{2 \alpha \gamma^{2}} \\
0 & e^{-\beta\left(\frac{k\left(a_{1}^{2} q_{1}-a_{0}^{2} q_{0}\right)\left(2 \alpha \gamma-N a_{1} q_{1}\right)}{8 \alpha \gamma^{2}}+\xi\right)}
\end{array}\right]
$$

so $E_{1}^{*}$ is stable along the eigendirection transverse to $r=1$ if $k>k_{1}$ with $k_{1}$ given by (18), and stable along the horizontal direction if $\frac{N a_{1} q_{1}}{2 \gamma}<\alpha<\frac{N a_{1} q_{1}}{2 \gamma}+2$.

Notice that all the stability conditions along the horizontal invariant lines $r=0$ and $r=1$, on which the boundary fixed points are located, correspond to those already examined for the logistic restrictions (9) and (12). Finally, at $E^{*}$ we have

$$
J\left(x^{*}, r^{*}\right)=\left[\begin{array}{cc}
1-\frac{\alpha x^{*}}{k} & -\frac{N\left(a_{1} q_{1}-a_{0} q_{0}\right) x^{*}}{2 \gamma} \\
\frac{\beta}{4 \gamma} r^{*}\left(1-r^{*}\right)\left(a_{1}^{2} q_{1}-a_{0}^{2} q_{0}\right) & 1
\end{array}\right]
$$

Hence, given that trace and determinant of the matrix (24) are respectively

$$
\begin{aligned}
\operatorname{Tr} & =2-\frac{\alpha x^{*}}{k}=2-\frac{4 \alpha \gamma \xi}{k\left(a_{0}^{2} q_{0}-a_{1}^{2} q_{1}\right)} \\
\text { Det } & =1-\frac{\alpha x^{*}}{k}+\frac{N \beta}{8 \gamma^{2}}\left(a_{1} q_{1}-a_{0} q_{0}\right)\left(a_{1}^{2} q_{1}-a_{0}^{2} q_{0}\right) x^{*} r^{*}\left(1-r^{*}\right)
\end{aligned}
$$


a sufficient condition for the local asymptotic stability of $E^{*}$ is that the eigenvalues of (24), which are solutions of the characteristic equation $P(z)=z^{2}-\operatorname{Tr} \cdot z+$ Det $=0$, are located inside the unit circle of the complex plane. A necessary and sufficient condition for this is given by the following system of inequalities (known as Schur or Jury's conditions, see e.g. [11])

$$
P(1)=1-\operatorname{Tr}+\text { Det }>0 ; P(-1)=1+\operatorname{Tr}+\text { Det }>0 ; \quad 1-\text { Det }>0
$$

In our case we have $P(1)=-\frac{4 N \beta \xi}{8 \gamma}\left(a_{1} q_{1}-a_{0} q_{0}\right) r^{*}\left(1-r^{*}\right)>0$ which vanishes (and then changes sign) when $r^{*}$ crosses the value $r^{*}=0$ from above and when $r^{*}$ crosses the value $r^{*}=1$ from below. These two conditions correspond to transcritical bifurcations when the interior equilibrium $E^{*}=\left(x^{*}, r^{*}\right)$ merges with the boundary points $E_{0}^{*}$ and $E_{1}^{*}$ respectively. In fact, the condition $r^{*}\left(x^{*}\right)=0$ implies $x^{*}=x_{0}^{*}$ and $r^{*}\left(x^{*}\right)=1$ implies $x^{*}=x_{1}^{*}$. The two bifurcation conditions can easily be expressed in terms of the carrying capacity $k$ as $k=k_{0}$ and $k=k_{1}$ respectively, see also Figure 1, where the equilibrium points are represented for $k$ in the range $\left[k_{0}, k_{1}\right]$. Notice that a change of sign of $P(1)$ also occurs when $\xi$ changes from negative to positive, and this is a transcritical bifurcation as well, but of codimension two as it occurs when the fixed point $E^{*}$ crosses at $\xi=0$ the segment of fixed points along the axis $x=0$.

Before analyzing the other two stability conditions, let us consider the condition $T r^{2}-4 D e t>0$ leading to real eigenvalues. This condition becomes

$$
2 \alpha^{2} \gamma^{2} x^{*}+16 k \alpha \gamma^{2}>k^{2} N \beta\left(a_{1} q_{1}-a_{0} q_{0}\right)\left(a_{1}^{2} q_{1}-a_{0}^{2} q_{0}\right) r^{*}\left(1-r^{*}\right)
$$

from which it is evident that it is surely satisfied (hence we have real eigenvalues) for $r^{*}$ very close to 0 or $r^{*}$ very close to 1 , whereas it is surely not satisfied (hence we have complex conjugate eigenvalues) for intermediate values of $r^{*}$ and sufficiently high values of the parameter $\beta$. Notice that both $x^{*}$ and $r^{*}$ do not depend on the parameter $\beta$.

Indeed, if the other parameters are fixed so that $x^{*}>0$ and $r^{*}$ has intermediate values, i.e. it is not too close to the invariant lines of pure strategies, then a Neimark-Sacker bifurcation occurs for increasing values of $\beta$. In fact, the third stability condition $1-$ Det $>0$, where a change of sign of the left hand side indicates the occurrence of a Neimark-Sacker bifurcation of $E^{*}$, becomes

$$
N \beta\left(a_{1} q_{1}-a_{0} q_{0}\right)\left(a_{1}^{2} q_{1}-a_{0}^{2} q_{0}\right) r^{*}\left(1-r^{*}\right)<\frac{8 \alpha \gamma^{2}}{k}
$$

This stability condition can be equivalently written as $\beta<\beta_{N S}$, with

$$
\beta_{N S}=\frac{8 \alpha \gamma^{2}}{N k\left(a_{1} q_{1}-a_{0} q_{0}\right)\left(a_{1}^{2} q_{1}-a_{0}^{2} q_{0}\right) r^{*}\left(1-r^{*}\right)}
$$

and $r^{*}$ given by (16), and a Neimark-Sacker bifurcation occurs when $\beta$ increases across $\beta_{N S}$.

Finally, the stability condition $P(-1)>0$ becomes

$$
32 k \gamma^{2}+N k \beta\left(a_{1} q_{1}-a_{0} q_{0}\right)\left(a_{1}^{2} q_{1}-a_{0}^{2} q_{0}\right) x^{*} r^{*}\left(1-r^{*}\right)>16 \alpha \gamma^{2} x^{*}
$$

that can be equivalently written as $\beta>\beta_{F}$ with

$$
\beta_{F}=2 \beta_{N S}-\frac{8 \gamma}{N \xi\left(a_{0} q_{0}-a_{1} q_{1}\right) r^{*}\left(1-r^{*}\right)}
$$

These results can be summarized by the following statement: 
Proposition 1 The map (7), with $\xi \in(-\infty,+\infty)$ and positive values of all other parameters, always has the boundary fixed points $E_{0}^{0}=(0,0)$ and $E_{1}^{0}=(0,1)$, and if $\xi=0$ any point of the whole segment $(0, r)$, with $r \in[0,1]$, is a fixed point. Moreover, the following holds:

- If $2 \alpha \gamma>N a_{i} q_{i}, i=0,1$, then two more boundary fixed points exist, namely $E_{0}^{*}=\left(x_{0}^{*}, 0\right)$ with $x_{0}^{*}$ given by $(10)$ and $E_{1}^{*}=\left(x_{1}^{*}, 1\right)$ with $x_{1}^{*}$ given by (13).

- If $\xi<0, a_{0}^{2} q_{0}<a_{1}^{2} q_{1}$ and $k_{0}<k<k_{1}$ where $k_{0}$ and $k_{1}$ are given by (17) and (18) respectively, then an interior equilibrium $E^{*}=\left(x^{*}, r^{*}\right)$ exists with components given by (16).

- If $E_{0}^{*}$ exists (i.e. $2 \alpha \gamma>N a_{0} q_{0}$ ) then for $\xi<0 E_{0}^{0}$ is a saddle point with stable set along the invariant line $x=0$ and unstable set along the invariant line $r=0$.

- If $E_{1}^{*}$ exists (i.e. $2 \alpha \gamma>N a_{1} q_{1}$ ) then for $\xi<0 E_{1}^{0}$ is an unstable node.

- $E_{0}^{*}$ is a stable node if $k<k_{0}$ and $\frac{N a_{0} q_{0}}{2 \gamma}<\alpha<\frac{N a_{0} q_{0}}{2 \gamma}+2$. At $k=k_{0}$ it undergoes a transcritical bifurcation at which it merges with $E^{*}$, at $\alpha<\frac{N a_{0} q_{0}}{2 \gamma}+2$ it undergoes a flip bifurcation along the invariant line $r=0$.

- $E_{1}^{*}$ is a stable node if $k>k_{1}$ and $\frac{N a_{1} q_{1}}{2 \gamma}<\alpha<\frac{N a_{1} q_{1}}{2 \gamma}+2$. At $k=k_{1}$ it undergoes a transcritical bifurcation at which it merges with $E^{*}$, at $\alpha<\frac{N a_{1} q_{1}}{2 \gamma}+2$ it undergoes a flip bifurcation along the invariant line $r=1$.

- The interior fixed point $E^{*}$ is stable if $k_{0}<k<k_{1}$ and $\beta_{F}<\beta<\beta_{N S}$, where $\beta_{N S}$ and $\beta_{F}$ are given by (30) and (32) respectively, hence the range of stability is nonempty provided that $\alpha \gamma \xi>-k\left(a_{1}^{2} q_{1}-a_{0}^{2} q_{0}\right)$

It is worth highlighting that condition $\beta_{F}<\beta_{N S}$ is equivalent to $k<\frac{\alpha x^{*}}{4}$ that, being $k>k_{0}$, is verified for $1+\alpha-\frac{N a_{0} q_{0}}{2 \gamma}<5$, that is true when the restriction (9) to $r=0$ has bounded dynamics, i.e. when $1+\alpha-\frac{N a_{0} q_{0}}{2 \gamma} \leq 4$. The stability range of the interior equilibrium $E^{*}$, as the parameter $\beta$ varies, is shown by the bifurcation diagrams in Figure 2, where the supercritical flip and Neimark-Sacker bifurcations, through which the equilibrium loses its stability for decreasing and increasing values of $\beta$ respectively, can be clearly seen.

\section{Global dynamics}

In this section we propose further analytical results and numerical explorations of some global dynamic scenarios of the discrete dynamical system (7) under the constraints on the parameters imposed by the economic and ecologic meaning of the model. The numerical simulations will confirm the analytical results on local stability and bifurcations given in the previous section and will give some snapshots about global dynamic behaviors.

Let us start by commenting the bifurcation diagrams presented in Figure 2. They show the long run dynamics of the model varying the intensity of choice parameter $\beta$, that represents the evolutionary propensity to switch to the more profitable technology. In evolutionary models a common occurrence is that an increase of the value of $\beta$ leads to instability and complex dynamics, 

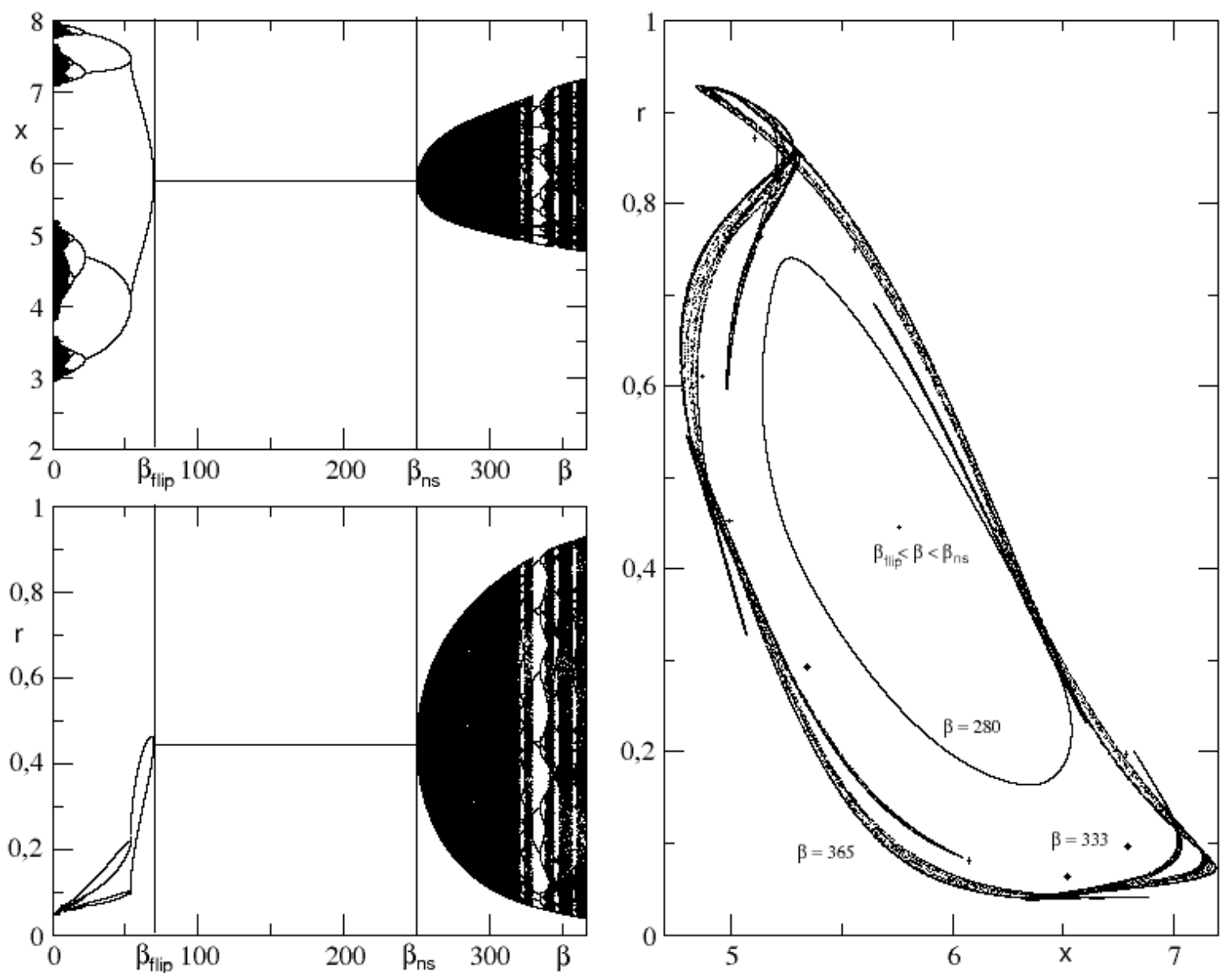

Figure 2: Left column: Bifurcation diagrams for $\beta \in[0,350]$ showing the asymptotic dynamics of the two state variables $x$ and $r$. Parameters: $\alpha=2.7, \gamma=1, N=15, a_{0}=1.05487, a_{1}=0.5$, $q_{0}=0.01, q_{1}=0.2, k=6.7$ and $\xi=-0.056$. Right column: Attractors on the $(x, r)$ phase space for different values of $\beta$. The other parameters are as in the left column.

see e.g. [14]. However, this is not necessarily true in the case of the evolutionary model studied herein. Indeed, starting from values close to zero, as $\beta$ increases we observe a transition from oscillatory dynamics (periodic or chaotic) towards non oscillatory dynamics through a cascade of period-halving bifurcations leading to the stability of the fixed point $E^{*}$. Moreover, we have a range of the values of $\beta$, namely $\beta \in\left(\beta_{F}, \beta_{N S}\right)$ as stated in Proposition 1 , such that the fixed point $E^{*}$ is stable. Furthermore, for $\beta>\beta_{N S}, E^{*}$ becomes unstable again through a supercritical Neimark-Sacker bifurcation, at which a stable invariant closed curve is created around $E^{*}$, whose amplitude increases as $\beta$ increases, see Figure 2 right panel. So, this example underlines the unusual result that low values of intensity of choice, as well as high values, lead to instability of the fixed point $E^{*}$ with the creation of periodic or quasi-periodic or even chaotic attractors, whereas intermediate levels of the intensity of choice are required for the local asymptotic stability of $E^{*}$.

Other interesting results regard the evolutionary dominance of one of the two harvesting strategies. On the basis of the analytical results in the previous section we know that as long as $E^{*}$ 
exists, then the two fixed points $E_{0}^{*}$ and $E_{1}^{*}$ are transversally unstable. At first sight, this may suggest that an evolutionary dominant strategy, or equivalently an attractor along the invariant lines $r=0$ or $r=1$, exits if and only if the interior fixed point $E^{*}$ is unfeasible and at the same time an attractor with $r \in(0,1)$ exits if and only if the interior fixed point $E^{*}$ is feasible. Instead, interior attractors (cyclic or chaotic) may exist even for $k>k_{1}$, i.e. after the transcritical bifurcation at which the equilibrium $E^{*}$ merges with $E_{1}^{*}$ and becomes unfeasible. Moreover, convergence towards the invariant line $r=0$ may occur even when the equilibrium $E^{*}$ is feasible, i.e. $k_{0}<k<k_{1}$. These two occurrences are stated by the following two Propositions, respectively.

Proposition 2 Consider map (7). Let $\xi<0$ and the other parameters are positive and such that $E_{1}^{*}$ exists and it is unstable along the manifold $r=1$, and a period-2 cycle, say $\mathcal{C}_{1}^{2}=$ $\left\{\left(x_{1}^{* 1}, 1\right),\left(x_{1}^{* 2}, 1\right)\right\}$, exists on the invariant set $r=1$, as the result of the period-doubling bifurcation of $E_{1}^{*}$. If the following condition holds

$$
\xi-\frac{k\left(a_{0}^{2} q_{0}-a_{1}^{2} q_{1}\right)}{2 \alpha \gamma}<\frac{k\left(a_{0}^{2} q_{0}-a_{1}^{2} q_{1}\right)\left(2 \gamma \alpha-N a_{1} q_{1}\right)}{8 \alpha \gamma^{2}}-\xi<0
$$

then the fixed point $E_{1}^{*}$ has stable transverse invariant manifold and period-2 cycle $\mathcal{C}_{1}^{2}$ is transversely unstable. The contrary cannot occur.

Proof. See Appendix A.

Proposition 2 hints at an interesting dynamic scenarios, confirmed by the numerical simulations shown in Figure 3. In this case, although there are no interior fixed points and the border equilibrium $E_{1}^{*}$ is transversely stable, the dynamics of the model can still converge in the long run to a stable inner attractor, a stable period-2 cycle $\mathcal{C}_{1}^{2}$ shown in Figure 3 (left panel). This evidence indicates that the stability of the transverse invariant manifold of $E_{1}^{*}$ does not imply the predominance by evolutionary pressure of the standard (or intensive) technology.

The situation is different (and in some sense reverted) when we consider the invariant line $r=0$, as stated in the following proposition.

Proposition 3 Consider map (7). Let $\xi<0$ and the other parameters are positive and such that $E_{0}^{*}$ exists and it is unstable along the invariant manifold $r=0$, and a period-2 cycle, say $\mathcal{C}_{0}^{2}=\left\{\left(x_{0}^{*_{1}}, 0\right),\left(x_{0}^{*_{2}}, 0\right)\right\}$, exists on the invariant set $r=0$, as the result of the period-doubling bifurcation of $E_{0}^{*}$. If the following condition holds

$$
\xi-\frac{k\left(a_{0}^{2} q_{0}-a_{1}^{2} q_{1}\right)}{2 \alpha \gamma}<\frac{k\left(a_{0}^{2} q_{0}-a_{1}^{2} q_{1}\right)\left(2 \gamma \alpha-N a_{0} q_{0}\right)}{8 \alpha \gamma^{2}}-\xi<0
$$

then the fixed point $E_{0}^{*}$ is transversely unstable and period-2 cycle $\mathcal{C}_{0}^{2}$ has a stable transverse invariant manifold. The contrary cannot occur.

Proof. See Appendix B.

The proposition 3 provides an interesting result highlighted by the numerical simulations shown in Figure 3 (right panel), where, although the border equilibrium $E_{0}^{*}$ is transversally unstable, the 
2-cycle $\mathcal{C}_{0}^{2}$ is locally asymptotically stable and coexists with the locally asymptotically stable interior fixed point $E^{*}$.

Numerical investigations suggest that this dynamic scenario occurs due to a specific sequence of bifurcations. In particular, a 2-cycle in the region with negative $r$, let us name it $\mathcal{C}^{2}$, undergoes a transcritical bifurcation, merging with the 2-cycle $\mathcal{C}_{0}^{2}$ originated by a period-doubling bifurcation of the fixed point $E_{0}^{*}$, and becomes feasible. After the bifurcation, $\mathcal{C}_{0}^{2}$ becomes local asymptotically stable while $\mathcal{C}^{2}$ is a saddle 2-cycle, and its one-dimensional stable manifold marks the boundary separating the basins of attraction of $E^{*}$ and $\mathcal{C}_{0}^{2}$. Then, changing the values of the parameters in a suitable way, the 2-cycle $\mathcal{C}^{2}$ disappears through a subcritical flip bifurcation at which $E^{*}$ looses stability and becomes a saddle fixed point. These bifurcations occur before the merging of $E^{*}$ with $E_{0}^{*}$. This underlines that agents can select the environmentally-friendly technology even when $E^{*}$ is a feasible fixed point and $E_{0}^{*}$ is transversely unstable. These results point out a quite peculiar property of the considered evolutionary model. In fact, the instability of the inner fixed point of the model may lead to an increase in the propensity of the agent to adopt the environmentallyfriendly technology. These scenarios do not occur on the continuous (or hybrid) setting of the model analyzed in [15].

The basins of attraction in Figure 3 underline further interesting properties of the dynamics of the model. In particular, from the right panel in Figure 3 it is possible to observe that the transverse unstable manifold of $E_{0}^{*}$ belongs to the basin of attraction of $E^{*}$, hence such a basin has a contact with the invariant line $r=0$ at the point $E_{0}^{*}$. This implies that all the preimages of $E_{0}^{*}$ along the invariant line $r=0$, computed according to the restriction (9), represent tongues at which the basin of $E^{*}$ has a contact with line $r=0$. In the figure only some of them are visible, but infinitely many exist and accumulate near $E_{0}^{0}$. The fine structure of these tongues is quite complicated and will be analyzed in future works.

It is worth observing that the mechanisms that lead to the evolutionary-dominant environmentallyfriendly technology when the fixed point $E^{*}$ is feasible, can be even different from the described one and, as shown in the following, can be due to the existence of non topological Milnor attractors on the invariant line $r=0$. For example, the bifurcation diagrams in Figure 4, obtained varying parameter $a_{0}$ in the range $[0.5,1.3]$, show another case such that an attracting invariant set $\mathcal{A}_{s}$, laying on the axis $r=0$, exists even when the interior equilibrium point $E^{*}$ is feasible. Measure-theoretic arguments about the transverse attractiveness of the invariant set $\mathcal{A}_{s}$ can be used to provide an explanation of this dynamic phenomenon. In particular, we make use of the transverse Lyapunov exponent (see e.g. [2], [8]) defined as:

$$
\Lambda_{\perp}=\lim _{N \rightarrow \infty} \frac{1}{N} \sum_{n=0}^{N} \ln \left|\nu_{\perp}\left(x_{n}\right)\right|
$$

where $\left\{x_{n}=f_{0}^{n}\left(x_{0}\right), n \geqslant 0\right\}$ is a trajectory embedded in $\mathcal{A}_{s}$ and $\nu_{\perp}\left(x_{n}\right)$ is the transverse eigenvalue computed in $x_{n}$. Precisely, when $\mathcal{A}_{s}$ is a $k$-cycle $\mathcal{C}_{0}^{k}$, its transverse attractiveness is measured by the product of the transverse eigenvalues $\nu_{\perp}^{k}=\prod_{i=1}^{k} \nu_{\perp}\left(x_{i}\right)$ and, if $\Lambda_{\perp}\left(\mathcal{C}^{k}\right)=k^{-1} \ln \left|\nu_{\perp}^{k}\right|<0$, then $\mathcal{A}_{s}$ is a topological attractor. Whilst, when the attractor $\mathcal{A}_{s}$ is chaotic and so includes infinitely many cycles densely distributed within it, each one characterized by its own transversal Lyapunov exponent, transverse attractiveness of $\mathcal{A}_{s}$ can be measured by the spectrum of the Lyapunov exponents. This is defined, see e.g. [8], as

$$
\Lambda_{\perp}^{\min } \leqslant \cdots \leqslant \Lambda_{\perp}^{\text {nat }} \leqslant \cdots \leqslant \Lambda_{\perp}^{\max }
$$



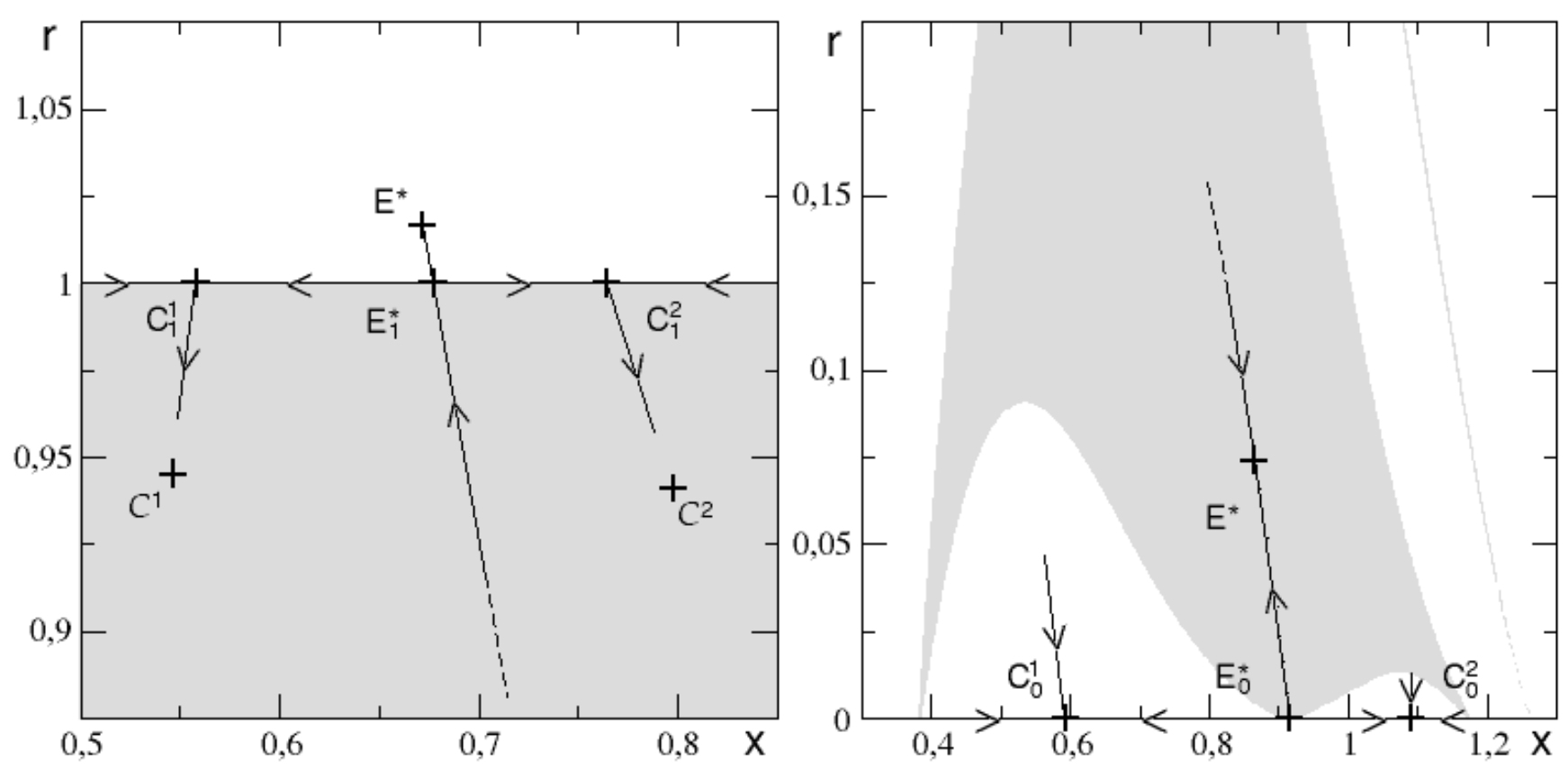

Figure 3: Left panel: The gray region is the basin of attraction of the inner period-2 cycle $\mathcal{C}_{1}^{2}$ while the fixed point $E_{1}^{*}$ is transversely attractive. The point $E^{*}$ is unfeasible, i.e. it lies in the region $r>1$. Parameters' values: $\alpha=3.1, k=1, N=8, q_{1}=1, q_{0}=0.01, \gamma=4, a_{0}=1.1$, $a_{1}=1, \beta=10, \xi=-0.0415$. Right panel: The gray region is the basin of attraction of the transversely stable fixed point $E^{*}$ and the white region is the basin of attraction of the period-2 cycle $\mathcal{C}_{0}^{2}$. Parameters' values: $\alpha=2.6, k=1, N=8, q_{1}=1, q_{0}=0.1, \gamma=2, a_{0}=1.1, a_{1}=1$, $\beta=80.615, \xi=-0.095$.

where $\Lambda_{\perp}^{\min }$ and $\Lambda_{\perp}^{\max }$ are the Lyapunov exponents of the most attractive and the most repelling cycles in $\mathcal{A}_{s}$ respectively. Moreover the natural Lyapunov exponent $\Lambda_{\perp}^{\text {nat }}$ is computed along a generic aperiodic trajectory embedded in $\mathcal{A}_{s}$, and it measures transversal attractiveness on average. In other words, $\Lambda_{\perp}^{\text {nat }}$ carries contributions to attractiveness from all the trajectories in $\mathcal{A}_{s}$, giving the mean local behavior in its neighborhood (see e.g. [19]). If $\mathcal{A}_{s}$ contains at least one transversely repelling cycle with a dense set of preimages embedded in $\mathcal{A}_{s}$, that is $\Lambda_{\perp}^{\max }>0$, than there is no neighborhood of $\mathcal{A}_{s}$ containing only points whose $\omega$-limit set belongs to $\mathcal{A}_{s}$. According to the theorem stated in [1] the latter inequality implies that the one-dimensional invariant chaotic set cannot be a Lyapunov attractor in the two-dimensional space because of the transversely unstable set of the period-2 cycle as well as its preimages. If the inequality $\Lambda_{\perp}^{\text {nat }}<0$ holds also, thus the set $\mathcal{A}_{s}$ attracts a positive measure set of points which converges to it. It follows that this is an attractor in Milnor sense (see [18]). A large number of results about global attractiveness of invariant manifold of lower dimension than the total phase space can be found in the literature, see e.g. [1], [2], [8].

In the right panel in Figure 4, we present both the transverse Lyapunov exponent characterizing transverse attractiveness of $\mathcal{A}_{s}$ and the transverse Lyapunov exponent of to the period-2 cycle $\mathcal{C}_{0}^{2}$. For suitable values of the aggregate parameter $\mu=1+\alpha-\frac{N}{2 \gamma} a_{0} q_{0}$, at which the 2-cycle 

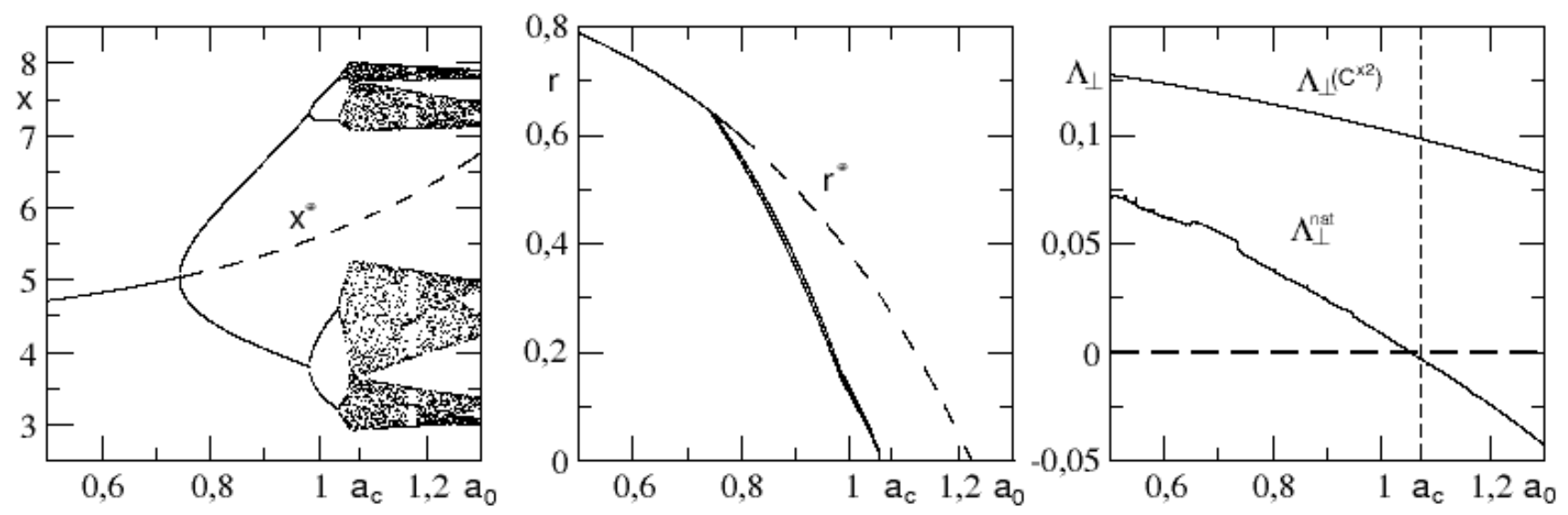

Figure 4: Bifurcation diagram of $x$ vs $a_{0}$ (left panel) and $r$ vs $a_{0}$ (center panel) varying $a_{0} \in$ $\left(a_{1}, a_{1} \sqrt{q_{1} / q_{0}}\right)$. Dashed lines represent paths of both $x^{*}$ and $r^{*}$. We can notice that the asymptotic dynamics is enclosed along the invariant axis $r=0$ even for $r^{*}\left(a_{0}\right)>0$. This is due to the transverse attractiveness of some subsets of the invariant axes $r=0$ while both the fixed point $E_{0}^{*}$ and the period- 2 cycle $\mathcal{C}_{0}^{2}$ are transversally repelling. For $\mu=1+\alpha-N a_{0} q_{0} / 2 \gamma \approx 3.5925721841$ such that $a_{0}=a_{c}=1.07428$, the attractor $\mathcal{A}_{s}$ of the logistic map on $r=0$ is characterized by pure chaos. Since $\Lambda_{\perp}^{\text {nat }}(\bar{\mu}) \approx-0.0034703<0$ while $\Lambda_{\perp}\left(\mathcal{C}_{0}^{2}\right)>0$, it results that $\mathcal{A}_{s}$ is a non topological Milnor attractor. Parameters' values are as in Figure 2 but $N=20$ and $\beta=5$.

of the logistic map undergoes the homoclinic bifurcation due to which 2-cyclic chaotic intervals are obtained by the merging of 4-cyclic chaotic intervals, pure chaos exists in $\mathcal{A}_{s}$ (see e.g [16]). For example, for $\alpha=2.7, N=20, \gamma=1, q_{0}=0.01$ and $a_{0}=a_{c}=1.07428$, we detect the presence of a Milnor attractor when $\mu=\bar{\mu} \approx 3.5925721841$, value at which $\Lambda_{\perp}^{\text {nat }}(\bar{\mu})<0$ while $\Lambda_{\perp}^{\max } \geqslant \Lambda_{\perp}\left(\mathcal{C}_{0}^{2}\right)>0$. Note that the parameter $\beta$ is the so called normal parameter, i.e. it affects only the transverse stability of $\mathcal{A}_{s}$ and does not have influence on the dynamics inside the invariant set $\mathcal{A}_{s}$. Setting $\mu=1+\alpha-\frac{N}{2 \gamma} a_{0} q_{0}=\bar{\mu}$ and varying $\beta$, we vary the spectrum of Lyapunov exponents, i.e. we change the topological property of the invariant set $\mathcal{A}_{s}$ which turns to be a chaotic saddle, a non topological Milnor attractor and a topological Lyapunov stable set.

To sum up we can say that $\mathcal{A}_{s}$ can attract a set of positive Lebesgue measures even when it contains repelling cycles together with dense sets of their preimages.

In the last part of the section, we point out that numerical simulations of the model can show dynamic scenarios which are difficult to infer analytically. For example, in Figure 5 we observe a chaotic attractor in the region $r \in(0,1)$ where both harvesting strategies coexist, although there is a prevalence of the environmentally-friendly one. The time series $r(t)$ shows an apparently stochastic behavior which typically characterizes the evolutionary dynamics driven by a replicator equation.

To conclude this section, we want to emphasize that the conducted investigation underlines that the class of evolutionary games, represented by the discrete-time model considered here, reveals some interesting and economically insightful dynamics, such as non topological Milnor attractors, that are not observable in other and more simple evolutionary games, see e.g. [14]. 

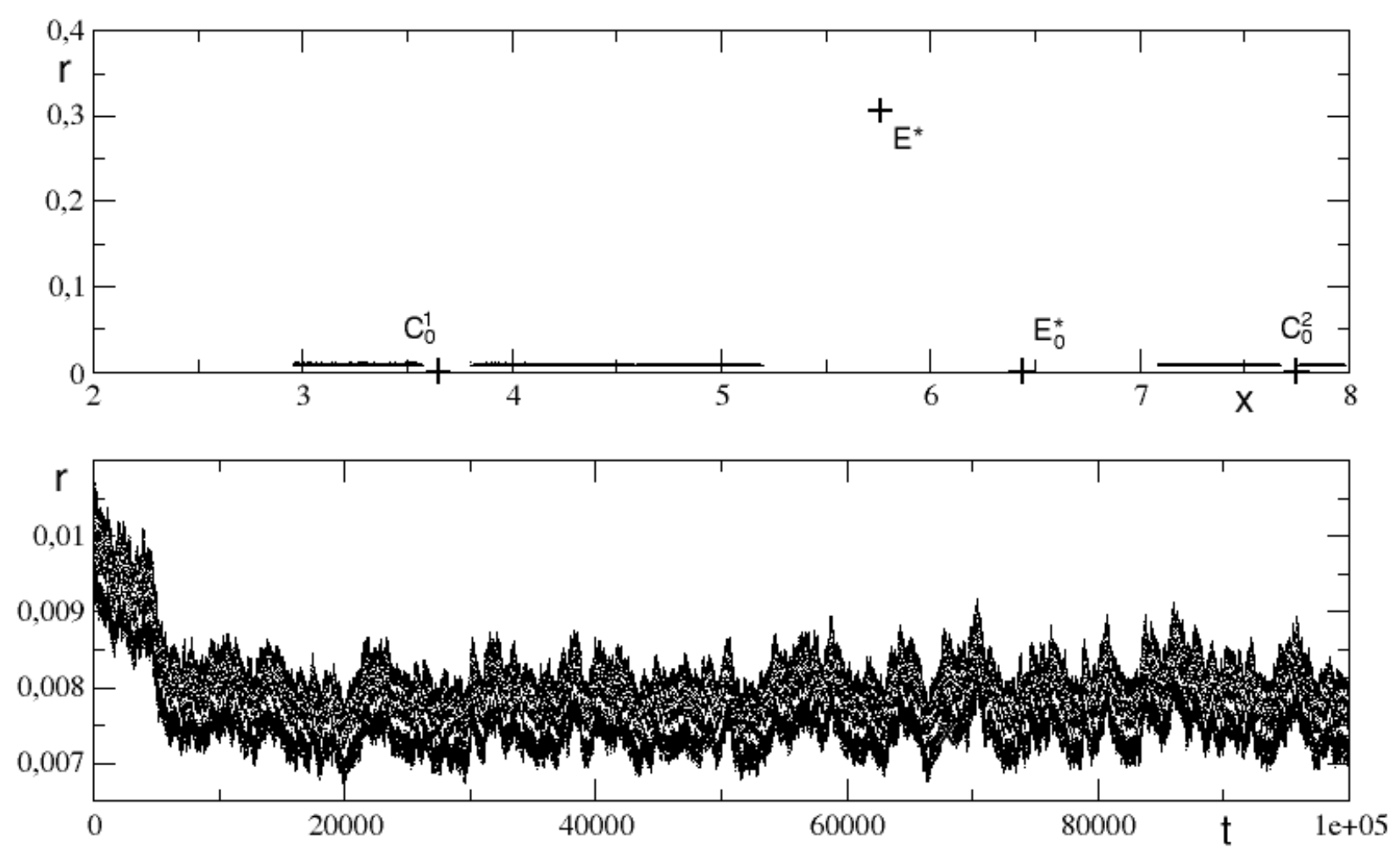

Figure 5: Upper panel: a trajectory in the phase space. Lower panel: a time series of $r(t)$. Parameters' values are as in Figure 2 but $N=20$ and $\beta=5$.

\section{Conclusions}

In this paper we have analyzed some local and global dynamical properties of a map that represents the discrete-time version of an evolutionary game model proposed in [15] to describe a fishery where a pool of fishermen can select between two different technologies of harvesting, that is an environmentally-friendly technology and a standard technology. The analysis of the dynamics underlines interesting scenarios that cannot occur on the continuous version of the model. In particular, Neimark-Sacker bifurcations and cascade of period-doubling bifurcations can lead to quite complicated, even chaotic, dynamics. Moreover, a deeper analytical and numerical analysis reveals the existence of Milnor attractors. Those attractors have interesting economic implications not only for the model proposed here, but for an entire class of evolutionary games that this model could represent. For this reason, the analysis and the presence of the Milnor attractors and related study of riddled basins for this class of evolutionary games, represent an interesting aspect that deserve further investigation.

Acknowledgments. We thank a Guest Editor and an anonymous Referee for useful comments and suggestions. This work has been performed within the framework of COST Action IS1104 "The EU in the new economic complex geography: models, tools and policy evaluation" and under the auspices of GNFM, Gruppo Nazionale di Fisica Matematica (Italy). 


\section{Appendix A}

Let us rewrite the map $T$ as follows:

$$
T:\left\{\begin{aligned}
x^{\prime} & =A x-B x^{2}+C x r \\
r^{\prime} & =\frac{r}{r+(1-r) e^{D x-\beta \xi}}
\end{aligned}\right.
$$

where

$$
A=1+\alpha-\frac{N a_{0} q_{0}}{2 \gamma} ; B=\frac{\alpha}{k} ; C=\frac{N}{2 \gamma}\left(a_{0} q_{0}-a_{1} q_{1}\right) ; D=\beta \frac{a_{0}^{2} q_{0}-a_{1}^{2} q_{1}}{4 \gamma}
$$

and let us consider the second iterate of the map $T$, i.e.

$$
T^{2}:\left\{\begin{aligned}
x^{\prime} & =\left(A x-B x^{2}+C x r\right)\left(A+C \frac{r}{r+(1-r) e^{D x-\beta \xi}}\right)-B\left(A x-B x^{2}+C x r\right)^{2} \\
r^{\prime} & =\frac{\frac{r}{r+(1-r) e^{D x-\beta \xi}}}{\frac{r}{r+(1-r) e^{D x-\beta \xi}}+\left(1-\frac{r}{r+(1-r) e^{D x-\beta \xi}}\right) e^{D\left(A x-B x^{2}+C x r\right)-\beta \xi}}
\end{aligned}\right.
$$

Its restriction to the invariant line $r=1$ is

$$
\left.T^{2}\right|_{r=1}:\left\{\begin{array}{l}
x^{\prime}=F\left(F x-B x^{2}\right)-B\left(F x-B x^{2}\right)^{2} \\
r^{\prime}=1
\end{array}\right.
$$

where

$$
F=A+C=1+\alpha-\frac{N a_{1} q_{1}}{2 \gamma}
$$

The map $T^{2}$ can have at most four fixed points, given by the solutions of the equation

$$
x\left(B^{3} x^{3}-2 F B^{2} x^{2}+F B(1+F) x+1-F^{2}\right)=0
$$

from which we obtain

$$
E_{1}^{0}=(0,1), E_{1}^{*}=\left(x_{1}^{*}, 1\right), E_{1}^{*_{1}}=\left(x_{1}^{* 1}, 1\right) \text { and } E_{1}^{*_{2}}=\left(x_{1}^{*_{2}}, 1\right)
$$

where $x_{1}^{*}$ is given by (13) and $x_{1}^{*_{1}}=\frac{1+F+\sqrt{F^{2}-3-2 F}}{2 B}, x_{1}^{*_{2}}=\frac{1+F-\sqrt{F^{2}-3-2 F}}{2 B}$.

Assuming the existence of period-2 cycle $\left\{\left(x_{1}^{* 1}, 1\right),\left(x_{1}^{* 2}, 1\right)\right\}$ of $T$ is equivalent to the existence of $E_{1}^{*_{1}}$ and $E_{1}^{*_{2}}$ for $T^{2}$ which, requiring also $x_{1}^{*_{1}}>0, x_{1}^{*_{2}}>0$, implies $F>3$, i.e. $\alpha-2>\frac{N a_{1} q_{1}}{2 \gamma}$. The Jacobian matrix associated to $T^{2}$ along the restriction $r=1$ is

$$
J^{2}(x, 1)=\left[\begin{array}{cc}
(F-2 B x)\left(F-2 B F x+2 B^{2} x^{2}\right) & J_{12}^{2}(x, 1) \\
0 & J_{22}^{2}(x, 1)
\end{array}\right]
$$

where $J_{22}^{2}(x, 1)=e^{D\left(F x-B x^{2}+x\right)-2 \beta \xi}$, from which we have the condition for transverse stability of $E_{1}^{* 1}$ and $E_{1}^{* 2}$, given by $J_{22}^{2}\left(x_{1}^{* 1}, 1\right)=J_{22}^{2}\left(x_{1}^{* 2}, 1\right)<1$. By trivial algebra we obtain the condition

$$
D\left(\frac{F+1}{B}\right)-2 \beta \xi<0
$$


Substituting for $D, F$ and $B$, we obtain

$$
\frac{k\left(a_{0}^{2} q_{0}-a_{1}^{2} q_{1}\right)\left(4 \gamma+2 \gamma \alpha-N a_{1} q_{1}\right)}{8 \alpha \gamma^{2}}-2 \xi<0
$$

By similar calculation, the condition to have stable the transverse manifold of the fixed point $E_{1}^{*}$ of $T^{2}$ is given by

$$
\frac{k\left(a_{0}^{2} q_{0}-a_{1}^{2} q_{1}\right)\left(2 \gamma \alpha-N a_{1} q_{1}\right)}{8 \alpha \gamma^{2}}-\xi<0
$$

From conditions (46) and (47) it follows that the transverse invariant manifold of $E_{1}^{*}$ is stable and the transverse invariant manifolds of $E_{1}^{*_{1}}$ and $E_{1}^{*_{2}}$ are unstable if and only if

$$
\frac{k\left(a_{0}^{2} q_{0}-a_{1}^{2} q_{1}\right)\left(4 \gamma+2 \gamma \alpha-N a_{1} q_{1}\right)}{8 \alpha \gamma^{2}}-2 \xi>0>\frac{k\left(a_{0}^{2} q_{0}-a_{1}^{2} q_{1}\right)\left(2 \gamma \alpha-N a_{1} q_{1}\right)}{8 \alpha \gamma^{2}}-\xi
$$

which can be rewritten as follows:

$$
\xi-\frac{k\left(a_{0}^{2} q_{0}-a_{1}^{2} q_{1}\right)}{2 \alpha \gamma}<\frac{k\left(a_{0}^{2} q_{0}-a_{1}^{2} q_{1}\right)\left(2 \gamma \alpha-N a_{1} q_{1}\right)}{8 \alpha \gamma^{2}}-\xi<0
$$

By simple considerations it is easy to note that conditions (49) and condition $\alpha-\frac{N a_{1} q_{1}}{2 \gamma}>2$ required for the existence of $E_{1}^{*_{1}}$ and $E_{1}^{*_{2}}$ identify a nonempty set of the parameter space.

Moreover, from stability condition (46) and (47) it is easy to note that in order to have the instability of the transverse invariant manifold of $E_{1}^{*}$ and the stability of the transverse invariant manifolds of $E_{1}^{*_{1}}$ and $E_{1}^{*_{2}}$ is required

$$
\frac{k\left(a_{0}^{2} q_{0}-a_{1}^{2} q_{1}\right)\left(4 \gamma+2 \gamma \alpha-N a_{1} q_{1}\right)}{8 \alpha \gamma^{2}}-2 \xi<0<\frac{k\left(a_{0}^{2} q_{0}-a_{1}^{2} q_{1}\right)\left(2 \gamma \alpha-N a_{1} q_{1}\right)}{8 \alpha \gamma^{2}}-\xi
$$

which can be rewritten as

$$
\frac{k\left(a_{0}^{2} q_{0}-a_{1}^{2} q_{1}\right)}{2 \alpha \gamma}-\xi<\xi-\frac{k\left(a_{0}^{2} q_{0}-a_{1}^{2} q_{1}\right)\left(2 \gamma \alpha-N a_{1} q_{1}\right)}{8 \alpha \gamma^{2}}<0
$$

Since throughout this paper we always assume $a_{0}^{2} q_{0}-a_{1}^{2} q_{1}<0$ and $\alpha-\frac{N a_{1} q_{1}}{2 \gamma}>2$ is required for the existence of $E_{1}^{*_{1}}$ and $E_{1}^{*_{2}}$, condition (51) implies that $(|\cdot|$ is the absolute value of $\cdot$ )

$$
|\xi|>\left|\frac{k\left(a_{0}^{2} q_{0}-a_{1}^{2} q_{1}\right)\left(2 \gamma \alpha-N a_{1} q_{1}\right)}{8 \alpha \gamma^{2}}\right|>\left|\frac{k\left(a_{0}^{2} q_{0}-a_{1}^{2} q_{1}\right)}{2 \alpha \gamma}\right|>|\xi|
$$

which is a contradiction. It follows that condition (51) cannot be satisfied.

Since the condition to have stable (or unstable) transversally manifold of the period 2-cycle $\left\{\left(x_{1}^{*_{1}}, 0\right),\left(x_{1}^{*_{2}}, 0\right)\right\}$ of the map $T$ is equivalent to condition to have stable transverse invariant manifold of each of the two fixed points $E_{1}^{*_{1}}$ and $E_{1}^{*_{2}}$ of $T^{2}$ and the condition to have stable (or unstable) transverse invariant manifold of the fixed point $E_{1}^{*}$ are the same for $T$ and $T^{2}$, the claim of the proposition follows. 


\section{Appendix B}

Let us consider the restriction of $T^{2}$, defined in Appendix A (see (37)), on the invariant line $r=0$

$$
\left.T^{2}\right|_{r=0}:\left\{\begin{array}{l}
x^{\prime}=A\left(A x-B x^{2}\right)-B\left(A x-B x^{2}\right)^{2} \\
r^{\prime}=0
\end{array}\right.
$$

It has at most four fixed points given by the solutions of the equation

$$
x\left(B^{3} x^{3}-2 B^{2} A x^{2}+A B(1+A) x+1-A^{2}\right)=0
$$

from which we obtain:

$$
E_{0}^{0}=(0,0), E_{0}^{*}=\left(x_{0}^{*}, 0\right), E_{0}^{*_{1}}=\left(x_{0}^{*_{1}}, 0\right) \text { and } E_{0}^{*_{2}}=\left(x_{0}^{*_{2}}, 0\right)
$$

where $x_{0}^{*}$ is given in (10), and $x_{0}^{* 1}=\frac{1+A+\sqrt{A^{2}-3-2 A}}{2 B}, x_{0}^{*_{2}}=\frac{1+A-\sqrt{A^{2}-3-2 A}}{2 B}$. Assuming the existence of period-2 cycle $\left\{\left(x_{0}^{*_{1}}, 0\right),\left(x_{0}^{*_{2}}, 0\right)\right\}$ of $T$ is equivalent to the existence of $E_{0}^{*_{1}}$ and $E_{0}^{*_{2}}$ for $T^{2}$ which, requiring also $x_{0}^{*_{1}}>0$ and $x_{0}^{*_{2}}>0$, implies $A>3$, i.e. $\alpha-2>\frac{N a_{0} q_{0}}{2 \gamma}$. The Jacobian matrix of $T^{2}$ along $r=0$ is

$$
J^{2}(x, 0)=\left[\begin{array}{cc}
(A-2 B x)\left(A-2 B A x+2 B^{2} x^{2}\right) & J_{12}^{2}(x, 0) \\
0 & J_{22}^{2}(x, 0)
\end{array}\right]
$$

where $J_{22}^{2}(x, 0)=e^{-D\left(A x-B x^{2}+x\right)+2 \beta \xi}$, from which the condition for transverse stability of $E_{0}^{*_{1}}$ and $E_{0}^{*_{2}}$ is $J_{22}^{2}\left(x_{0}^{*_{1}}, 0\right)=J_{22}^{2}\left(x_{0}^{*_{2}}, 0\right)<1$. By trivial algebra we obtain the condition

$$
D\left(\frac{1+A}{B}\right)-2 \beta \xi>0
$$

Substituting for $D, A$ and $B$, we obtain

$$
\frac{k\left(a_{0}^{2} q_{0}-a_{1}^{2} q_{1}\right)\left(4 \gamma+2 \gamma \alpha-N a_{0} q_{0}\right)}{8 \alpha \gamma^{2}}-2 \xi>0
$$

By similar calculation, the condition for transverse stability of the fixed point $E_{0}^{*}$ of $T^{2}$ is given by

$$
\frac{k\left(a_{0}^{2} q_{0}-a_{1}^{2} q_{1}\right)\left(2 \gamma \alpha-N a_{0} q_{0}\right)}{8 \alpha \gamma^{2}}-\xi>0
$$

From the conditions (58) and (59) it follows that transverse invariant manifold of $E_{0}^{*}$ is unstable and transverse invariant manifolds of $E_{0}^{*_{1}}$ and $E_{0}^{*_{2}}$ are stable if and only if

$$
\frac{k\left(a_{0}^{2} q_{0}-a_{1}^{2} q_{1}\right)\left(2 \gamma \alpha-N a_{0} q_{0}\right)}{8 \alpha \gamma^{2}}-\xi<0<\frac{k\left(a_{0}^{2} q_{0}-a_{1}^{2} q_{1}\right)\left(4 \gamma+2 \gamma \alpha-N a_{0} q_{0}\right)}{8 \alpha \gamma^{2}}-2 \xi
$$

which can be rewritten as follows

$$
\xi-\frac{k\left(a_{0}^{2} q_{0}-a_{1}^{2} q_{1}\right)}{2 \alpha \gamma}<\frac{k\left(a_{0}^{2} q_{0}-a_{1}^{2} q_{1}\right)\left(2 \gamma \alpha-N a_{0} q_{0}\right)}{8 \alpha \gamma^{2}}-\xi<0
$$


By simple considerations it is easy to note that conditions (61) and condition $\alpha-\frac{N a_{0} q_{0}}{2 \gamma}>2$ required for the existence of $E_{0}^{*_{1}}$ and $E_{0}^{*_{2}}$ identify a nonempty set of the parameter space.

Moreover, from stability condition (58) and (59) it is easy to note that in order to have the stability of the transverse invariant manifold of $E_{0}^{*}$ and the instability of the transverse invariant manifolds of $E_{0}^{*_{1}}$ and $E_{0}^{*_{2}}$ is required

$$
\frac{k\left(a_{0}^{2} q_{0}-a_{1}^{2} q_{1}\right)\left(2 \gamma \alpha-N a_{0} q_{0}\right)}{8 \alpha \gamma^{2}}-\xi>0>\frac{k\left(a_{0}^{2} q_{0}-a_{1}^{2} q_{1}\right)\left(4 \gamma+2 \gamma \alpha-N a_{0} q_{0}\right)}{8 \alpha \gamma^{2}}-2 \xi
$$

which can be rewritten as follows

$$
0>-\frac{k\left(a_{0}^{2} q_{0}-a_{1}^{2} q_{1}\right)\left(2 \gamma \alpha-N a_{0} q_{0}\right)}{8 \alpha \gamma^{2}}+\xi>\frac{k\left(a_{0}^{2} q_{0}-a_{1}^{2} q_{1}\right)}{2 \alpha \gamma}-\xi
$$

Since throughout this paper we always assume $a_{0}^{2} q_{0}-a_{1}^{2} q_{1}<0$ and $\alpha-\frac{N a_{0} q_{0}}{2 \gamma}>2$ is required for the existence of $E_{0}^{*_{1}}$ and $E_{0}^{*_{2}}$, condition (63) implies that $(|\cdot|$ is the absolute value of $\cdot)$

$$
|\xi|>\left|\frac{k\left(a_{0}^{2} q_{0}-a_{1}^{2} q_{1}\right)\left(2 \gamma \alpha-N a_{0} q_{0}\right)}{8 \alpha \gamma^{2}}\right|>\left|\frac{k\left(a_{0}^{2} q_{0}-a_{1}^{2} q_{1}\right)}{2 \alpha \gamma}\right|>|\xi|
$$

which is a contradiction. It follows that condition (63) cannot be satisfied.

Since the condition to have stable (or unstable) the transverse invariant manifold of the two period cycle $\left\{\left(x_{0}^{*_{1}}, 0\right),\left(x_{0}^{*_{2}}, 0\right)\right\}$ of the map $T$ is equivalent to condition to have stable (or unstable) the transverse invariant manifold of each of the two fixed points $E_{0}^{*_{1}}$ and $E_{0}^{*_{2}}$ of $T^{2}$ and the condition to have stable (or unstable) the transverse invariant manifold of the fixed point $E_{0}^{*}$ are the same for $T$ and $T^{2}$, the claim of the proposition follows.

\section{References}

[1] J. C. Alexander, J. A. Yorke, Z. You, and I. Kan. Riddled basins. International Journal of Bifurcation and Chaos, 2(4):795-813, 1992.

[2] P. Ashwin, J. Buescu, and I. Stewart. From attractor to chaotic saddle: a tale of transverse instability. Nonlinearity, 9(3):703-737, 1996.

[3] G. I. Bischi, F. Lamantia, and D. Radi. Multi-species exploitation with evolutionary switching of harvesting strategies. Natural Resource Modeling, 26(4):546-571, 2013.

[4] G. I. Bischi, F. Lamantia, and D. Radi. A prey-predator model with endogenous harvesting strategy switching. Applied Mathematics and Computation, 219(20):10123-10142, 2013.

[5] G. I. Bischi, F. Lamantia, and L. Sbragia. Competition and cooperation in natural resources exploitation: an evolutionary game approach. In: Carraro, C., Fragnelli, V. (Eds.), Game Practice and the Environment, edward elgar publishing edition, 2009.

[6] G. I. Bischi, F. Lamantia, and F. Tramontana. Sliding and oscillations in fisheries with on-off harvesting and different switching times. Communications in Nonlinear Science and Numerical Simulation, 19(1):216-229, 2014. 
[7] F Brauer and C. Castillo-Chavez. Mathematical Models in Population Biology and Epidemiology, volume Texts in Applied Mathematics 40. Springer-Verlag, New York, 2001.

[8] J. Buescu. Exotic Attractors. Birkh'auser, Basel, 1997.

[9] A. Cabrales and J. Sobel. On the limit points of discrete selection dynamics. Journal of Economic Theory, 57(2):407-419, 1992.

[10] J. M. Cushing. Some discrete competition models and the competitive exclusion principle. Journal of Difference Equations and Applications, 10(13-15):1139-1151, 2004.

[11] S. N. Elaydi. An introduction to difference equations. New York, Springer, 1995.

[12] S. A.H. Geritz and E. Kisdi. On the mechanistic underpinning of discrete-time population models with complex dynamics. Journal of Theoretical Biology, 228(2):261-269, 2004.

[13] W. M. Getz and R.G. Haight. Population Harvesting: Demographic Models of Fish, Forest, and Animal Resources. Princeton University Press, 1989.

[14] J. Hofbauer and K. Sigmund. Evolutionary game dynamics. Bulletin (New Series) of the American Mathematical Society, 40(4):479-519, 2003.

[15] F. Lamantia and D. Radi. Exploitation of renewable resources with differentiated technologies: an evolutionary analysis. Mathematics and Computers in Simulation, 108:155-174, 2015.

[16] Y. L. Maistrenko, V. L. Maistrenko, A. Popovich, and E. Mosekilde. Transverse instability and riddled basins in a system of two coupled logistic maps. Physical Review E, 57(3):2713$2724,1998$.

[17] R. M. May. Biological populations obeying difference equations: stable points, stable cycles, and chaos. Journal of Theoretical Biology, 51(2):511-524, 1975.

[18] J. Milnor. On the concept of attractor: Correction and remarks. Communications in Mathematical Physics, 102(3):517-519, 1985.

[19] Y. Nagai and Y.-C. Lai. Periodic-orbit theory of the blowout bifurcation. Physical Review E, 56(4):4031-4041, 1997.

[20] P. Taylor and L. Jonker. Evolutionarily stable strategies and game dynamics. Mathematical Biosciences, 40(1):145-156, 1978. 